\title{
Pivotal response treatment for autism spectrum disorder: current perspectives
}

This article was published in the following Dove Press journal:

Neuropsychiatric Disease and Treatment

20 June 2017

Number of times this article has been viewed

Jiedi Lei

Pamela Ventola

Yale Child Study Center, Yale University School of Medicine, New Haven, CT, USA
Correspondence: Pamela Ventola Yale Child Study Center, Yale University School of Medicine, 230 South Frontage Road, PO Box 207900, New Haven, CT 06519 , USA

Tel + I 2037355657

Email pamela.ventola@yale.edu

\begin{abstract}
Pivotal response treatment (PRT) is an evidence-based behavioral intervention based on applied behavior analysis principles aimed to improve social communication skills in individuals with autism spectrum disorder (ASD). PRT adopts a more naturalistic approach and focuses on using a number of strategies to help increase children's motivation during intervention. Since its conceptualization, PRT has received much empirical support for eliciting therapeutic gains in greater use of functional social communication skills in individuals with ASD. Building upon the empirical evidence supporting PRT, recent advancements have increasingly turned to using interdisciplinary research integrating neuroimaging techniques and behavioral measures to help identify objective biomarkers of treatment, which have two primary purposes. First, neuroimaging results can help characterize how PRT may elicit change, and facilitate partitioning of the heterogeneous profiles of neural mechanisms underlying similar profile of behavioral changes observed over PRT. Second, neuroimaging provides an objective means to both map and track how biomarkers may serve as reliable and sensitive predictors of responder profiles to PRT, assisting clinicians to identify who will most likely benefit from PRT. Together, a better understanding of both mechanisms of change and predictors of responder profile will help PRT to serve as a more precise and targeted intervention for individuals with ASD, thus moving towards the goal of precision medicine and improving quality of care. This review focuses on the recent emerging neuroimaging evidences supporting PRT, offering current perspectives on the importance of interdisciplinary research to help clinicians better understand how PRT works and predict who will respond to PRT.
\end{abstract}

Keywords: PRT, ASD, biomarkers, neuroimaging, precision medicine, biological motion

\section{History of autism spectrum disorder and behavioral treatments}

Autism, from the Greek "autos" meaning self, was first poignantly captured by Kanner $^{1}$ in his lucid account of Donald T, a child who experienced significant social impairments. Donald was described to be so "self-satisfied"1 that "to get his attention almost requires one to break down a mental barrier between his inner consciousness and the outside world". ${ }^{1}$ Today, autism spectrum disorder (ASD) is still perceived as a pervasive developmental disorder, characterized by social communication deficit and narrow interest in objects and repetitive behavior. ${ }^{2}$ Up to one-third of young children with ASD also experience clinically significant levels of maladaptive behaviors, such as withdrawal, inattention, and aggression. ${ }^{3}$ Early behavioral interventions in the 1970s that aimed to increase social communication and reduce clinically significant maladaptive behaviors heavily relied on the use of operant conditioning principles, ${ }^{4}$ the most influential model being applied behavioral analysis (ABA). ${ }^{5}$ 
Traditional ABA aimed to elicit behavioral modifications through highly intensive and structured trials, where adultchosen stimuli are repeatedly presented to induce target behaviors in individuals with ASD, with correct responses reinforced. 5,6 More recently, advancements in ABA have led to more individualized and comprehensive treatments to target a wide range of adaptive behaviors that follow a more natural and normal developmental sequence, in children with ASD as young as three to four years of age. ${ }^{7,8}$ Although ABA has been demonstrated to be effective in both improving social functioning and reducing clinically significant maladaptive behaviors, ${ }^{5,6,9,10}$ clinical progress can be costly in both time and effort, which has received much criticism. ${ }^{6,10,11}$ First, individuals often experience increased exposure to failed attempts on highly structured trials, which can further decrease motivation, ${ }^{12}$ and induce a sense of learned helplessness. ${ }^{13}$ Second, highly structured training bears limited ecological validity, further compromising the generalizability of any successfully acquired skills across other developmental domains, as well as outside of clinical settings. ${ }^{6,14,15}$

\section{A brief outline of pivotal response treatment}

Pivotal response treatment (PRT) is a behavioral intervention aimed to improve social communication skills in individuals with ASD, ${ }^{10,11,16,17}$ which has accumulated a large evidence base with positive findings being replicated using a wide range of experimental designs across multiple settings. ${ }^{17}$ Based on ABA principles, PRT adopts a more naturalistic approach that focuses on targeting skills that are pivotal to development across social, communication, and behavior. ${ }^{11,18}$ "Pivotal" refers to a set of targeted skills which, when successfully acquired, can elicit more widespread positive clinical gains in the child's other domains of functioning. ${ }^{18}$ Some pivotal areas identified include motivation, self-initiation, and selfmanagement, which have been shown to be critical in eliciting broader improvements across multiple developmental domains, thus maximizing treatment gains. ${ }^{11,18,19}$

PRT focuses on using a number of strategies to help increase children's motivation during intervention, such as using a variety of child-chosen activities that are intrinsically motivating to each child, ${ }^{12,20}$ as well as interspersing maintenance and acquisition tasks to strengthen children's exposure to well-established response-reinforcer contingency. ${ }^{21,22}$ Similar to ABA, the structure of PRT involves the presentation of repeated behavioral trials consisting of antecedent, behavior, and consequence, ${ }^{15,23}$ where the antecedent presents clear opportunities prompting the child for a desired behavior. In contrast to traditional ABA, PRT reinforces both correct behavioral responses and any valid attempts made by the child en route to skill acquisition, thus increasing frequency of exposure to response-reinforcement contingency, in order to help maintain and increase child's motivation throughout the intervention. ${ }^{20,24}$

\section{PRT - empirical evidence and questions to address}

Since its original conception, PRT has received much empirical support for eliciting therapeutic gains for promoting greater use of functional social communication skills in individuals with ASD, ranging from increased self-initiated social responses ${ }^{25,26}$ to advancing collateral language acquisition following increased question-asking behavior. ${ }^{19,27,28}$ Increased adaptive use of language and social responses have also been linked to secondary clinical gains such as reduced disruptive behavior, ${ }^{29}$ and restrictive and repetitive behaviors. ${ }^{30}$ Such secondary clinical gains further support that targeting skills such as increasing social motivation and initiation of appropriate social responses may indeed be pivotal in securing changes in other behavioral domains that are less explicitly addressed during PRT intervention. ${ }^{17,30}$ For example, the use of child-preferred activities that carry high intrinsic motivational salience to children with ASD may be especially beneficial for providing opportunities to elicit joint attention, ${ }^{31}$ as well as teaching children to engage in symbolic play. ${ }^{32}$ Joint attention and pretend play are both crucial social skills underlying the emergence of higher-order social cognition, such as perspective taking and theory of mind development, ${ }^{33-37}$ and pivotal for securing children's competence at navigating social situations.

Building upon the empirical evidence supporting PRT, recent advancements have increasingly turned to using interdisciplinary research integrating neuroimaging techniques and behavioral measures to help identify biomarkers of treatment. ${ }^{38-40}$ The identification of sensitive and objective biomarkers serves two primary goals. First, neuroimaging facilitates better characterization of the heterogeneous profiles of neural mechanisms underlying behavioral changes observed over PRT, further increasing the evidence base surrounding how PRT may be eliciting changes across individuals with ASD. ${ }^{38,39}$ Second, mapping and tracking biomarkers across the course of development and intervention can instigate the generation of predictive models of responder profiles to PRT, thus assisting clinicians to identify who will most likely benefit from PRT in order to maximize 
therapeutic gains. ${ }^{40}$ Together, a better understanding of both mechanisms of change and predictors of responder profile will help PRT to serve as a more precise and targeted intervention for individuals with ASD, thus moving towards the goal of precision medicine and improving quality of care. ${ }^{41}$

The purpose of the current review is to review the recent emerging evidence from interdisciplinary perspective integrating behavioral and neuroscience research. Specifically, the current review examines (1) identification of neural biomarkers that objectively elucidate the underlying mechanisms of change following PRT and (2) behavioral and neural predictors of responder profile to PRT among individuals with ASD. Summaries of relevant studies are outlined in Table 1. Broader issues including limitations on the research quality supporting PRT, such as determining effective intervention study designs to help monitor change as well as conducting long-term follow-up, are discussed, with directions for future research outlined.

\section{From behavior to neuroscience: identifying mechanisms of change}

Although PRT has received much empirical support as an intervention targeting core social communication deficits in individuals with ASD from behavioral studies, few studies have investigated the possible underlying mechanisms and pathways that have elicited the changes observed. ${ }^{38}$ From a clinical perspective, reliance on observable behavioral changes as the sole outcome measure often suffers from the problem of equifinality. ${ }^{42}$ More precisely, similar profiles in behavioral changes could mask heterogeneous underlying mechanisms of change that are pivotal in eliciting therapeutic gains observed. Overcoming the problem of equifinality has important underlying rationales. Characterizing differential mechanisms of change underlying similar behavioral profiles can help clinicians understand how a child may be specifically benefitting from the intervention. Distilling down the key treatment components that can successfully tackle a specific behavioral profile can be especially informative for formulating treatment plans. Multiple strategies can be devised based around these crucial therapeutic components tailored to each child, generating a more focused and direct intervention plan that may be more cost-effective and time-efficient. Generating greater evidence highlighting differential mechanisms of treatment response can therefore guide clinicians to make evidence-based choices when reformulating treatment strategies. This is in line with moving towards the goal of precision medicine, where it is no longer sufficient to gather empirical evidence supporting if an intervention is working. The aim is to better partition how the intervention is working for each patient given his or her complex clinical profile. ${ }^{41}$

Developing objective and sensitive biomarkers is crucial for helping clinicians to understand the range of clinical profiles that are likely to benefit from the same intervention via differential mechanisms of change. For PRT, recent advancements have focused on investigating whether neural markers for social cognition identified from neuroimaging studies can serve as reliable biomarkers to assist quantification, and characterization, of differential mechanisms of change associated with social gains following PRT. ${ }^{38,39,43,44}$ One well-validated paradigm in search for neural correlates of social cognition and perception is the biological motion paradigm, which uses point-light displays that contain sufficient information to depict social movements such as a person walking, dancing, or playing games familiar to children such as pat-a-cake (Figure 1). ${ }^{43,45,46}$ Biological motion capitalizes on the intrinsic preferential visual sensitivity found in typically developing (TD) children and adults to movements that are biologically coherent rather than scrambled motion, which is an early emerging mechanism that serves to bias one's attention towards perceiving stimuli that carry greater social salience. ${ }^{46,47}$ Preferential bias towards biological motion perception is an important precursor to the emergence of higher-level social cognition ${ }^{34,48}$ and affect regulation, ${ }^{49}$ and thus serves as an important behavioral marker for social development.

To identify brain correlates relating to biological motion perception, Kaiser et $\mathrm{al}^{43}$ compared and contrasted children with ASD ( $\mathrm{n}=25)$ with their unaffected siblings (US; $\mathrm{n}=20$ ) and TD children $(n=17)$ to determine patterns of brain response that can serve as neural signatures underpinning social cognition differences in individuals with ASD when watching biological motion versus scrambled motion using functional magnetic resonance imaging (fMRI). The authors conducted group comparisons examining the effects of biological motion versus scrambled motion as follows: TD versus ASD, TD versus US, US versus ASD, and US versus TD; whole-brain analyses were conducted with activations noted at a voxel-wise uncorrected threshold level of $P<0.05$. To correct for multiple comparisons, clusters that exceeded the cluster threshold of $k>20$ contiguous voxels at $\alpha<0.05$ from each group comparison were selected. Setting the cluster threshold at $k>20$ contiguous active voxels within each group contrast resulted in a $<5 \%$ false-positive discovery rate of active voxels in the key group contrasts. The relative frequency of occurrence for each cluster size during 


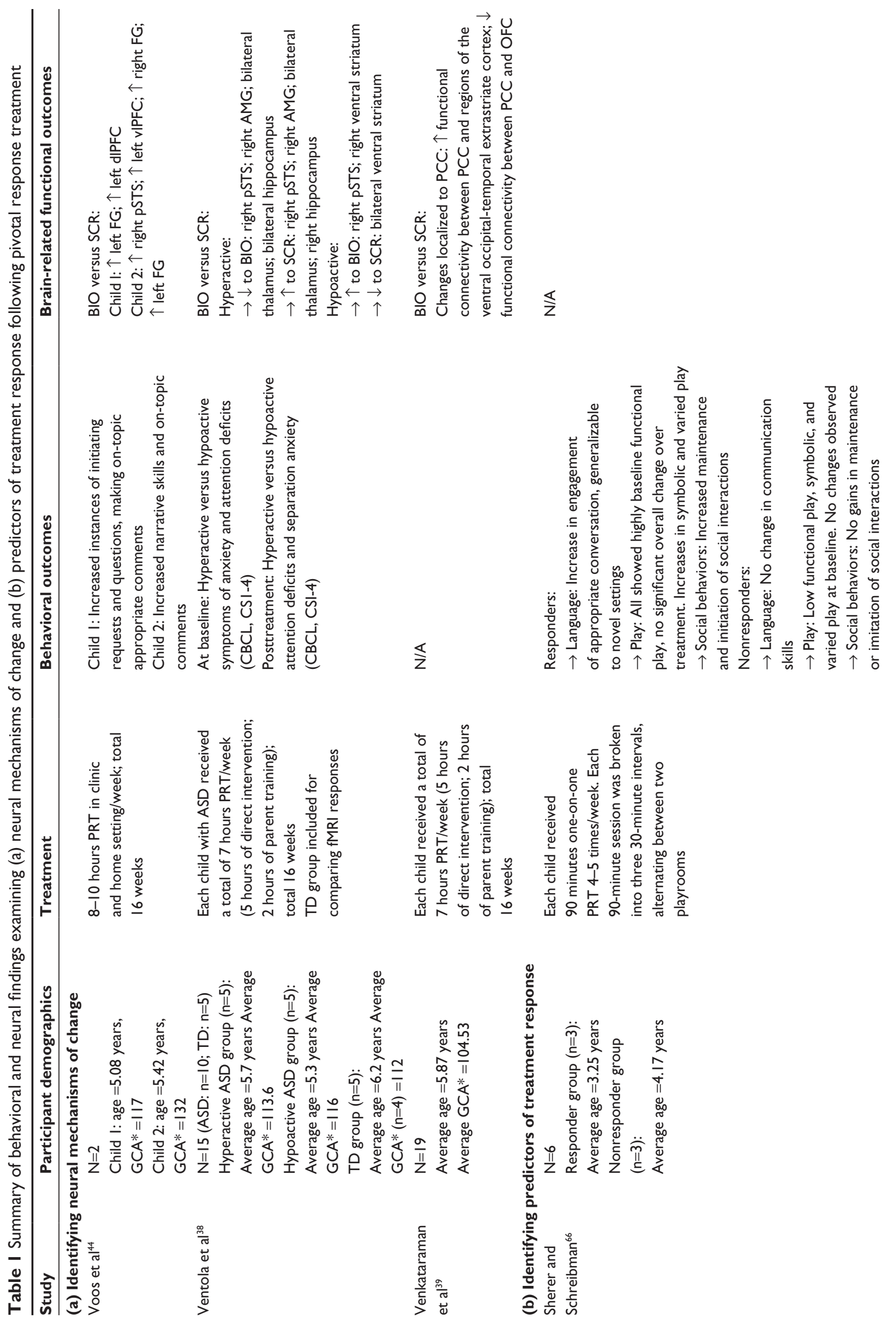



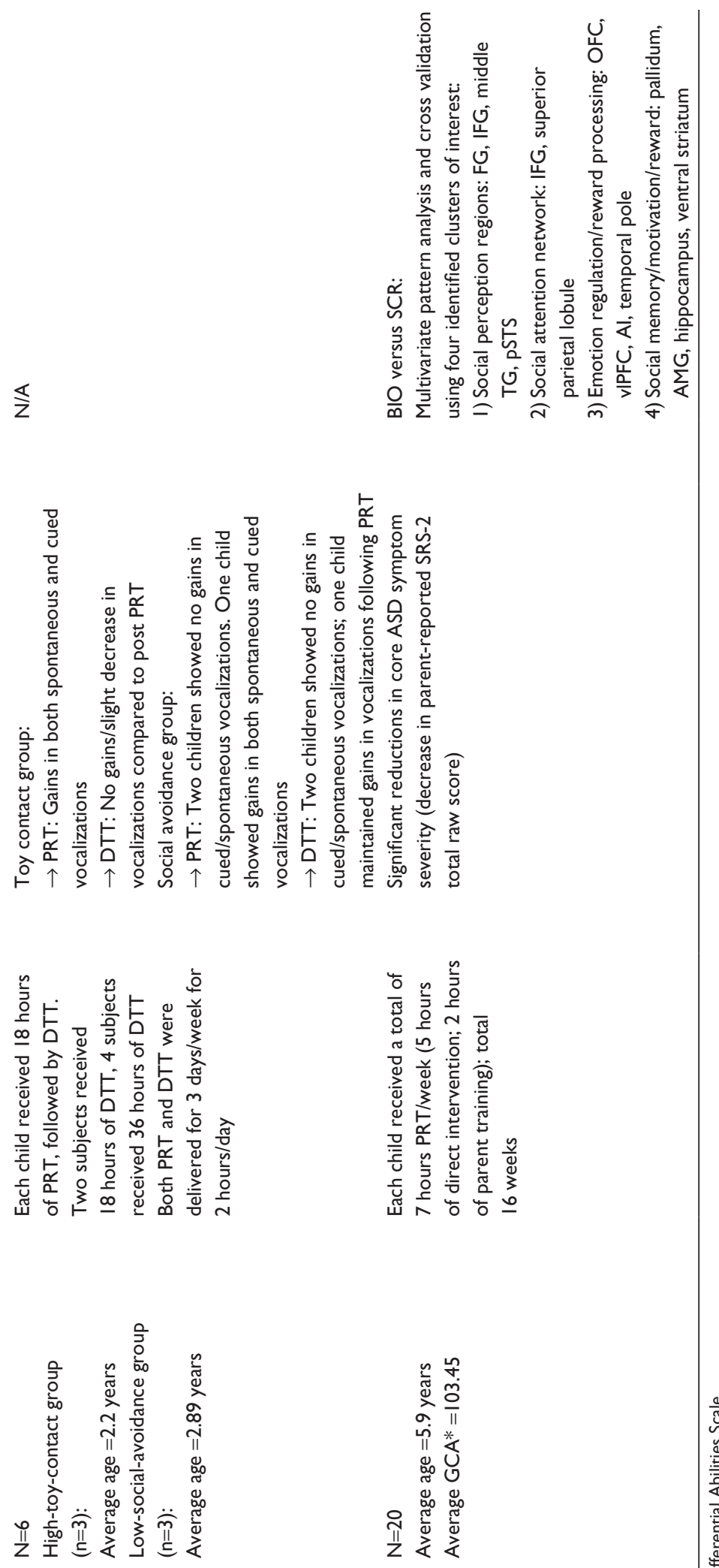

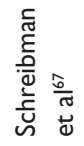
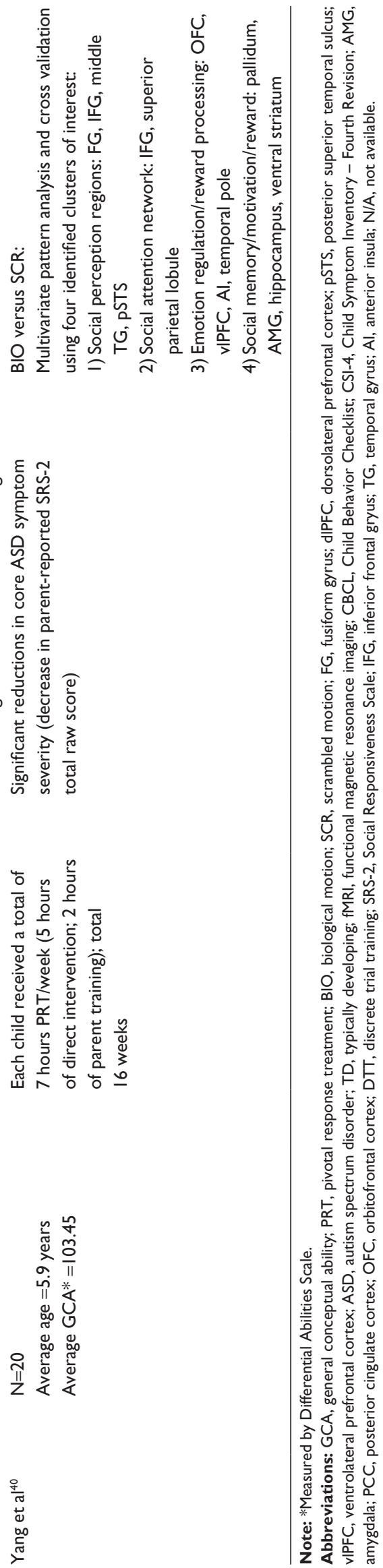


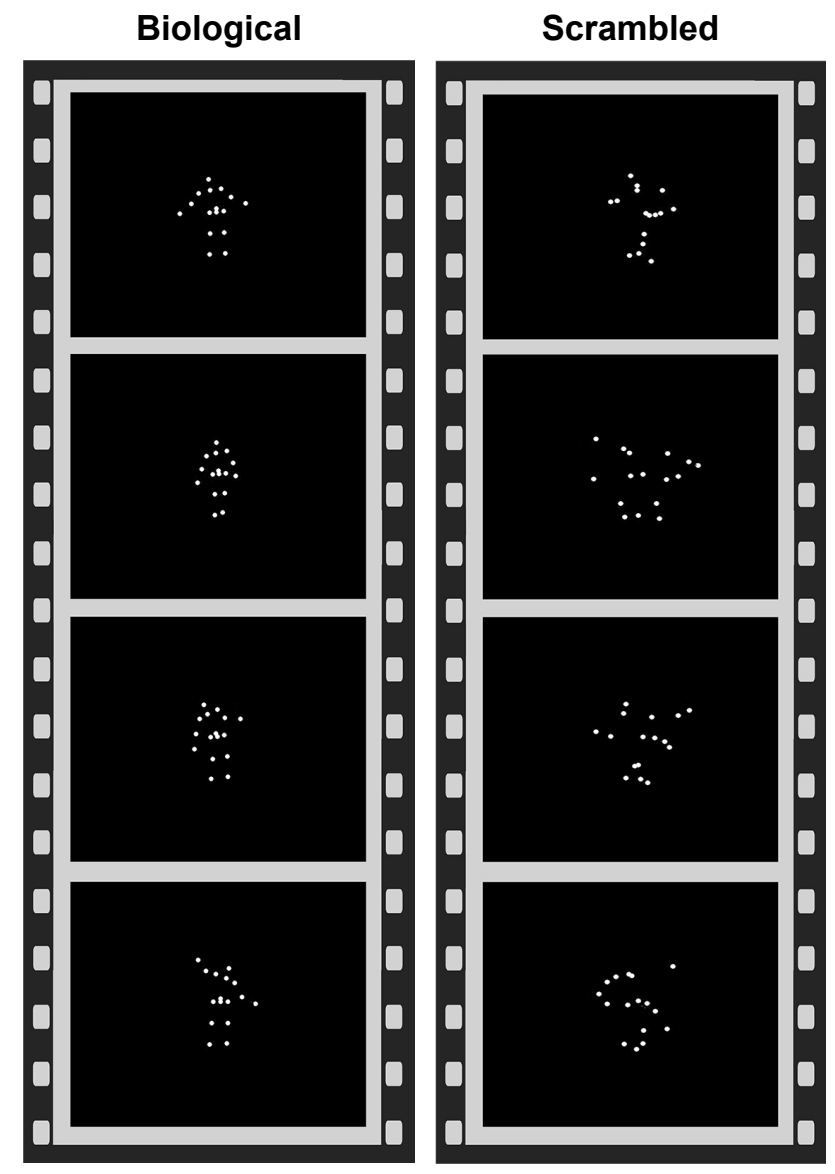

Figure I Point-light displays showing biological versus scrambled motion paradigm.

5,000 iterations using Monte Carlo simulation helped to inform assignment of $\alpha$ value for each cluster. Higher-level conjunction analyses to identify state, trait, and compensatory regions were conducted at the voxel-wise uncorrected threshold level of $P<0.0025$, and a cluster threshold of $k>20$, to further limit the rate of false positives in the final result output. The authors distinguished distinct patterns of (a) "state", (b) "trait", and (c) "compensatory" brain response to biological motion (Figure 2).

"State" regions demonstrated diminished activation in response to biological versus scrambled motion unique to ASD. "State" profile reflects disrupted brain processing of social stimuli specifically associated with the psychopathological state of ASD. "State" regions included left ventrolateral prefrontal cortex (vlPFC), ventromedial prefrontal cortex (vmPFC), right amygdala, right posterior superior temporal sulcus (rpSTS), and bilateral fusiform gyrus (FG). "Trait" regions demonstrated diminished activation in response to biological motion for both individuals with ASD and US relative to TD children. "Trait" profile may indicate possible disruption to social stimuli processing associated with shared genetic vulnerability between individuals with ASD and US, and a marker for endophenotype of ASD. "Trait" regions included left dorsolateral prefrontal cortex, right inferior temporal gyrus, and anterior bilateral FG. "Compensatory" regions demonstrated unique profile of activation to biological motion found in US group only, which may serve to overcome increased vulnerability to developing social perception deficits in US. "Compensatory" regions included caudal rpSTS and anterior rostral vmPFC. Taken together, the neural profiles identified included many brain areas that are associated with social responsiveness, mentalizing, and theory of mind..$^{50-52}$

The "state", "trait", and "compensatory" neural profiles therefore serve as a guide for identifying biomarkers that are sensitive to differences in perception of social stimuli across children with ASD, US, and TD children. Using sensitive biomarkers to monitor changes in patterns of neural activity in response to biological motion provides an objective means to quantify whether behavioral changes in social cognition over the course of PRT are accompanied by underlying functional neural changes. Specifically, based on positive social gains from behavioral measures following PRT, one may hypothesize that individuals with ASD may also show increased brain activation when perceiving biological motion, thus normalizing attention bias towards socially salient stimuli. ${ }^{44}$ Characterizing whether changes in brain response to biological motion perception may occur in "state" or "compensatory" regions may be especially informative for understanding how PRT may help improve social functioning in individuals with ASD. For example, improved social functioning may be driven by either increased normalization of developmental trajectory of social cognition in children with ASD compared to TD children (increased activation of "state" and "trait" regions to biological motion) or an increased recruitment of compensatory regions to overcome social perception difficulties similar to US, or both.

Voos et $\mathrm{a}^{44}$ conducted the first fMRI study to evaluate changes in brain activation in response to biological motion following an open-label trial of 16 weeks of PRT in two high-functioning preschool-age children with ASD. The authors found that both children showed increased activation in "state" and "trait" regions in response to biological motion posttreatment, though distinct yet overlapping neural profiles emerged, which the authors concluded to be somewhat reflective of the level of social skills targeted for each child. For participant 1, goals were set to target lowerlevel adaptive social skills such as body positioning and voice modulation. For participant 2, goals were set to target 

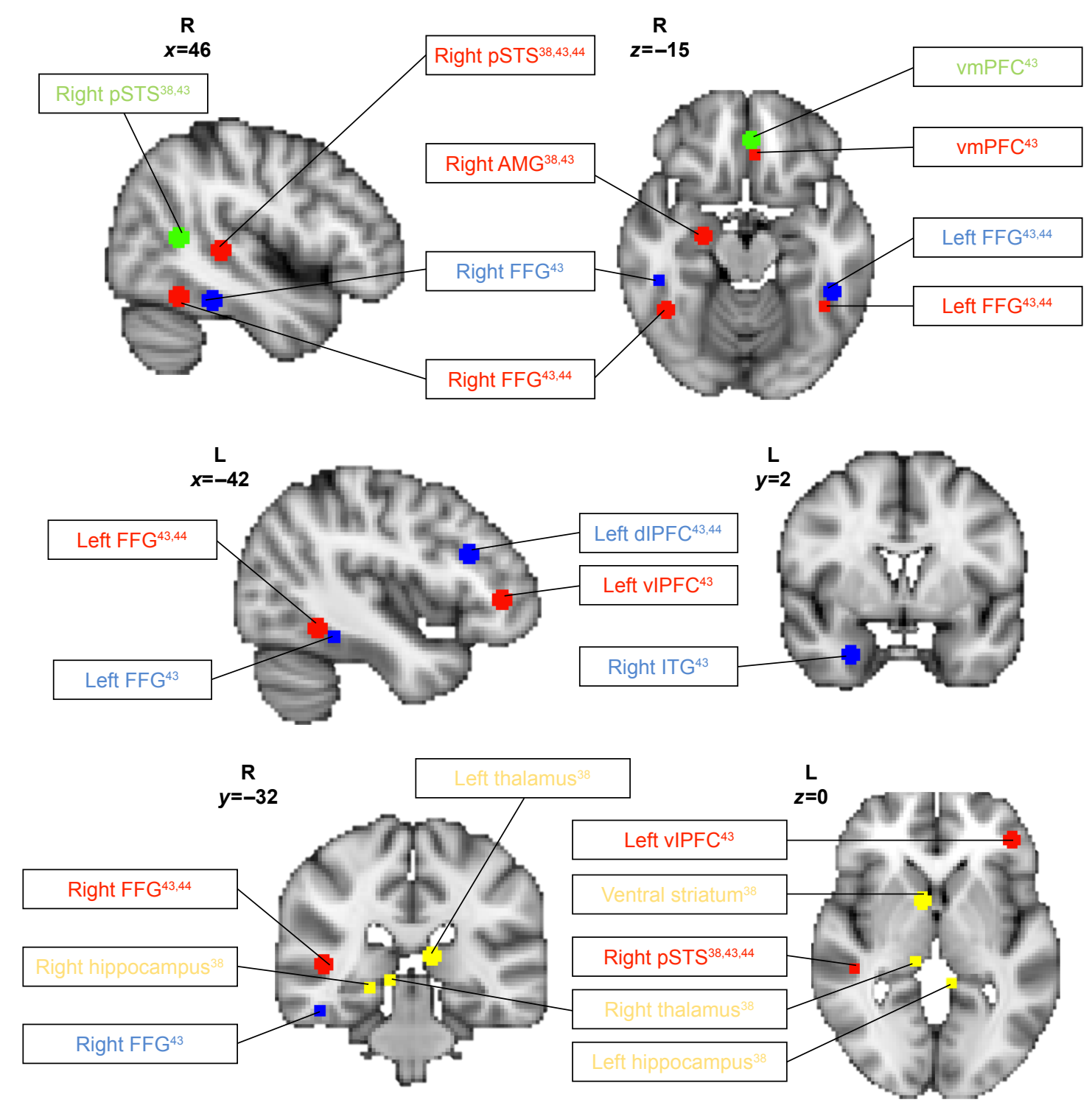

State regions ${ }^{43} \quad$ Trait regions $^{43} \quad$ Compensatory regions $^{43} \quad$ Other regions $^{38}$

Figure 2 Brain regions demonstrating differences in activation during biological motion relative to scrambled motion.

Notes: State (red), trait (blue), and compensatory (green) regions were identified by Kaiser et al, ${ }^{43}$ and many similar areas showed changes in activation in response to biological motion in Voos et al's study. ${ }^{44}$ Additional regions that showed changes in activation to biological motion as identified by Ventola et al ${ }^{38}$ are listed in yellow.

Abbreviations: pSTS, posterior superior temporal sulcus; AMG, amygdala; FFG, facial fusiform gyrus; vmPFC, ventromedial prefrontal cortex; dIPFC, dorsolateral prefrontal cortex; ITG, inferior temporal gyrus; vIPFC, ventrolateral prefrontal cortex.

higher-level social skills such as perspective taking, social reciprocity, and adaptive use of descriptive language. Both children made significant gains in adaptive social functioning as measured by both the Autism Diagnostic Observation Schedule ${ }^{53}$ and the Clinical Evaluation of Language Fundamentals Fourth Edition, ${ }^{54}$ and demonstrated increased activation in bilateral FG ("state") in response to biological motion, suggesting increased sensitivity to socially salient stimuli following PRT. However, only participant 2 demonstrated more widespread increased activation in state-defined rpSTS and vlPFC, which have been associated with higher-level social processing ${ }^{55}$ such as perspective taking and understanding intention of others ${ }^{56}$ which may directly reflect the more advanced nature of social skills directly targeted during his intervention (Figure 2). In contrast, no changes in "compensatory" regions were observed.

Given the small sample size of two, findings generated from Voos et al's $s^{44}$ study were largely preliminary, and could only offer a limited scope for interpretation. Nonetheless, the findings were promising and are important for 
future studies to consider due to three main reasons. First, behavioral clinical gains corresponded to brain activation changes in response to biological motion, suggesting that neural changes might serve as a sensitive and objective biomarker for measuring changes in social functioning over PRT. Second, the increased activation to biological motion was localized in "state" and "trait" regions only, suggesting that PRT may serve to normalize the developmental trajectory of neural mechanisms of social stimuli processing in children with ASD, by increasing recruitment of brain regions associated with social perception in TD children. Finally, the overlapping, yet distinct patterns of changes in brain activation following PRT across both participants further highlight the value of neuroimaging techniques to partition differential patterns of neural change underlying behavioral changes, and mark a step towards resolving the problem of equifinality. However, the small sample size of two children severely limits the ability to draw firm conclusions that can be generalized to a larger clinical population, especially to those of lower-functioning children with ASD. Furthermore, the lack of control group makes it unclear whether the neural changes observed were directly related to the therapeutic effects of PRT, some more general effects of development over the 16-week time window, or a combination.

Another way to directly address the issue of equifinality is to capitalize on the idea that heterogeneous behavioral and neural response profiles at baseline may undergo differential trajectories of change following PRT. Heterogeneity at baseline can thus serve as a marker for dividing participants into meaningful groups, where subsequent changes over the course of PRT can be directly compared and contrasted to evaluate differences in mechanisms of change. Utilizing fMRI and the biological motion paradigm, Ventola et $\mathrm{al}^{38}$ compared and contrasted the baseline neural response to biological versus scrambled motion in rpSTS of each of 10 high-functioning school-aged children with ASD to the group average response of five TD children. Two distinct patterns of neural responses were identified: a "hyperactive" group (hyperactive rpSTS activation) and a "hypoactive" group (hypoactive rpSTS activation), relative to TD group average. The authors hypothesized that the hyperactive group may experience increased sensitivity to external stimuli, as a result of deficits in neural systems responsible for sensory gating (eg, increased activation in thalamus, amygdala, temporal cortical regions), and effective attention control. ${ }^{57}$ In contrast, the hypoactive group may experience diminished social motivation, as a result of deficits in neural systems responsible for processing reward in response to social stimuli $^{58}$ (eg, reduced activation in nucleus accumbens, ventral striatum [VS], and amygdala). Despite distinct neural profiles in response to biological motion at baseline, both groups presented similar levels of reduced attention to faces and poor eye contact at baseline, thus highlighting the importance of using neuroimaging tools as an objective way of capturing underlying heterogeneity in the absence of distinct behavioral markers.

Following PRT, distinct neural patterns of mechanisms of change across the two groups emerged. ${ }^{38}$ Given that PRT primarily targets social communication skills and appropriate response to social stimuli, the authors examined changes in response to biological versus scrambled motion that were driven primarily by differential response to biological motion pre- and post-PRT. The hyperactive group demonstrated reduced activation in rpSTS, amygdala, thalamus, and hippocampus in response to biological motion following PRT, as well as reduced functional connectivity between left amygdala and left prefrontal regions. For the hyperactive group, PRT may have elicited positive gains in neural processing of sensory gating and attention control. In contrast, the hypoactive group demonstrated increased activation in rpSTS and VS in response to biological motion following PRT, and significant increases in functional connectivity between bilateral VS and pSTS as well as nodes of the mirror neuron system (eg, right inferior frontal gyrus, precentral cingulate) (Figure 2). For the hypoactive group, PRT may have elicited positive gains in neural processing of reward in response to socially meaningful stimuli. It is important to highlight that these distinct mechanisms of change were accompanied by considerable clinical gains as well as "normalized" patterns of response to perceiving socially meaningful stimuli, further suggesting that individuals may benefit from PRT in different ways in terms of increasing both self-regulation and social cognition. Translating back to clinical work, distinct neural responses may serve as objective biomarkers that help clinicians to identify and better monitor different therapeutic elements of PRT at work, an especially powerful tool when used in combination with behavioral data and subjective questionnaire ratings to better monitor how PRT may elicit positive therapeutic gains across a wider range of responder profiles.

Building upon positive gains in normalizing trajectories of functional connectivity in neural circuits underlying reduced social motivation and inefficient sensory gating and attention control, ${ }^{38}$ it is important to investigate how PRT may influence neural circuitries involved in processing socially meaningful stimuli at a global level. ${ }^{39}$ The nature of social 
cognition deficits in individuals with ASD may arise from multiple levels of social information processing, ranging from the coordination of social motivation and the ability to attune one's attention to socially meaningful stimuli, ${ }^{58,59}$ to the ability to recognize and interpret perceived social stimuli such as biological motion and faces for higher-level processing. ${ }^{46,47,60}$ In a recent study, Venkataraman et al ${ }^{39}$ analyzed brain response to biological motion using fMRI and found that PRT might prompt changes in functional connectivity in social processing circuitry. The authors adopted a data-driven approach and estimated that posterior cingulate cortex (PCC), an area involved in both selfregulation and social cognition, ${ }^{61-63}$ is a locus representing aggregated functional change in response to biological motion among 19 high-functioning school-aged children with ASD following PRT. Changes in PCC activity were accompanied by both decreased functional synchrony to orbitofrontal cortex, implicated in evaluating reward value associated with external stimuli, ${ }^{64}$ and increased functional synchrony to occipital-temporal cortex, implicated in perception of biological motion and facial recognition. ${ }^{65}$ The authors interpreted this shift in functional synchrony as a result of PRT prompting increased recognition of socially meaningful stimuli, and engagement in higher-order social perception areas.

However, a major limitation of Venkataraman et al's $\mathrm{s}^{39}$ study is the lack of control group, since neither a TD group nor a waitlist control group was included for comparison. It thus remains unclear whether changes in functional synchrony observed may be elicited by PRT per se rather than reflecting naturally occurring changes over the course of development among individuals with ASD. Furthermore, one cannot assess whether the quality of changes in functional synchrony may represent a closer alignment to that seen during social information processing found in typical development. Nonetheless, this study represents a promising first step towards the use of data-driven approach to investigate changes in functional synchrony at a brain circuitry level in response to social information following PRT.

\section{From behavior to neuroscience: identifying responder profiles and predictors of change}

Another important area critical to improving quality of care for children and young people with ASD is to characterize responder profiles to the treatment in question. The benefit of developing objective biomarkers that may help clinicians predict treatment outcome at baseline is twofold.
First, such biomarkers can help clinicians select the most appropriate form of intervention to maximize treatment gain for the individual in question. Given that there are a wide variety of behavioral-based interventions for children with ASD, identifying predictors that are specific to the active ingredients of PRT is important, in order to aid clinicians assess whether the fit of PRT is appropriate for each child. Second, identifying behavioral and brain response profiles of nonresponders at baseline can indicate problem areas that are more treatment resistant, and guide clinicians to be more mindful of these issues when formulating treatment plans and identifying specific goals. Being more mindful, clinicians may consider implementing strategies to provide additional support for these more challenging and persistent issues, as well as taking into account how such adaptations may influence fidelity of treatment. To our knowledge, only three studies up to date have evaluated predictors of treatment outcome in children with ASD in response to PRT, identifying a mixture of behavioral and brain response biomarkers to characterize responder profile. Here, we summarize each study, highlighting the limitations arising from the paucity of literature in this area.

Using a retrospective design, Sherer and Schreibman ${ }^{66}$ compared the baseline profiles of six children with ASD (average age 3-4 years) who received 90-minute sessions of PRT, four to five times per week, for 5-6 months. Three children showed significant improvements and were characterized as responders to PRT, and three children did not show any significant change and were characterized as nonresponders to PRT. The children were matched for IQ, use of functional language, and autism symptom severity at baseline. The study aimed to examine whether differences in social behavior and play skills at baseline served as behavioral markers predicting treatment outcome. Both social and play skills were assessed using a child-mother interaction social probe, where mothers were given specific instructions to initiate language, reciprocal play, and responding to requests from their child over 5-minute intervals. Social behaviors included both initiation of interaction and the ability to sustain interaction. Play behaviors included functional use of toys, symbolic and pretend play, and the ability to engage in varied play. The authors found that compared to nonresponders, all three responders showed greater engagement in functional, symbolic, and varied play at baseline, and experienced improvements in all three areas over the course of PRT. In addition, responders also showed significant gains in ability to sustain social engagement following PRT. In contrast, nonresponders demonstrated lower levels 
of baseline play and social skills compared to responders, and saw little change in these functional behaviors over the course of PRT.

Given the well-matched cognitive, language, and autism symptom severity profiles at baseline, differences in therapeutic gains over the course of PRT could not be attributed to differences in cognitive capacity or language and social competency. It may be that those who displayed poorer social and play skills at baseline experienced a floor effect. PRT primarily serves to increase social communication skills through utilizing varied play activities that are naturally motivating for children, ${ }^{20,21}$ and creating opportunities naturally embedded in social interactions for teaching appropriate social responses. Therefore, such a floor effect in both play skills and social reciprocity at baseline might compromise individuals' ability to learn through play during PRT, affecting long-term prognosis. However, given the small sample size and lack of alternative treatment for comparison, it is unclear whether such nonresponder profiles and the possible floor effects of low baseline social engagement may be a predictor of poor outcome following PRT only, or represent a generalized floor effect impacting treatment response to behavioral interventions in general. Finally, the identified responder and nonresponder profiles included a complex range of social and play-based behaviors, therefore making it difficult to identify whether there may be a specific active predictor that may account for a majority of variance associated with treatment outcome.

To address the question of treatment specificity, Schreibman et al ${ }^{67}$ employed a prospective design by recruiting six children with ASD (age 2-4 years) who matched the nonresponder behavior profile identified from the above study at baseline, and compared intervention outcome following PRT and an alternative behavioral training known as discrete trial training (DTT). Compared to PRT, DTT is a highly structured behavioral intervention where target behavior is broken down into smaller components, each of which is taught using repeated discrete trials. ${ }^{14,16}$ All children received 18 hours of PRT first, followed by 18-36 hours of DTT. To identify whether poor treatment response may be related to either social avoidance or play-based skills measured at baseline, the authors identified that the six participants were matched to the nonresponder profile with three children demonstrating high toy contact and three children demonstrating low social avoidance at baseline.

The authors found that the nonresponder profile only predicted treatment outcome of PRT, and not that of DTT, suggesting PRT specificity. Furthermore, children who demonstrated high toy contact at baseline showed some minimal, yet relative greater, response to PRT compared to the group with low social avoidance. Degree of social avoidance and contact at baseline did not predict treatment response differences, suggesting that having low social avoidance at baseline may be neither necessary nor sufficient to help children respond to PRT. There are several limitations to this study. First, all children received PRT first, and therefore, it is unclear whether any therapeutic potentials following subsequent DTT may in fact reflect either delayed response to PRT or the result of cumulative benefits from prior PRT in combination with DTT. ${ }^{67}$ Second, with a small sample size of three children in each group, it cannot be concluded whether interest in objects and toy contact measured at baseline may be a necessary factor for eliciting positive treatment response following PRT.

One way to partition how each behavioral predictor identified at baseline may account for variances in changes elicited over the course of PRT ${ }^{66,67}$ is to identify objective biomarkers such as neural clusters that can serve as correlates of baseline behaviors such as motivation, social responsiveness, and attention. ${ }^{40}$ Differential baseline neurobiological responsiveness to social stimuli in each distinct neural cluster can serve as an objective proxy measure of how much its behavioral correlate may affect an individual's overall social cognition capacity. So far, only one study has identified neurobiological markers that can predict treatment response to PRT. Using biological motion paradigm and fMRI, Yang et $\mathrm{al}^{40}$ identified four neural clusters whose activation pattern during biological motion at baseline predicted treatment gains in social competency following 16 weeks of PRT in 20 children with ASD (mean age 5.9 years). Structurally, the clusters included areas such as the STS, superior parietal lobule, FG, vmPFC, VS, and putamen. Functionally, the clusters corresponded to a range of lower- and higherlevel cognitive processes, ranging from motion and object perception, visuospatial attention, emotion regulation, and response inhibition, to reward and motivation.

The authors employed regression-based multivariate pattern analyses (MVPAs) to examine how unique activation patterns of voxels within neural networks may contribute to the function and efficiency of complex cognitive processes. ${ }^{40}$ Using cross-validation design, MVPA modeling of pretreatment response to biological motion trained using one cohort of patients' data significantly predicted changes in social impairment severity following PRT in an independent second cohort of patients' data, thus illustrating that the biomarkers identified were both robust and generalizable 
across patient samples. However, the lack of comparison treatment group makes it difficult to conclude whether the neurobiological biomarkers identified were predictors specific to PRT, rather than a general neural profile indicative of brain's readiness to respond to behavioral-based social skills interventions. Nonetheless, this study demonstrated an important first step toward using novel neuroimaging analyses to identify sensitive and robust biomarkers to predict treatment outcomes.

It is important to consider the value of such biomarkers in combination with individuals' behavioral profiles, to gain a better understanding of how certain neurobiological profiles may be associated with behavioral predictors such as social avoidance and toy contact. ${ }^{66,67}$ Techniques such as MVPAs provide a more sensitive means to detect inter-participant variations in neurobiological response to social stimuli at baseline, and provide a useful means to help partition heterogeneity underlying similar behavioral manifestations at baseline to further increase precision when predicting response to treatment. Therefore, it is important to consider the complementary value of employing interdisciplinary research techniques, such as qualitative behavioral measures and neuroimaging analyses, when moving towards the goal of precision medicine. ${ }^{41}$ One limitation of the current preliminary findings is the lack of information on broader systemic influences such as the type of educational settings the participants were in, socioeconomic status, and other environmental factors that may have played a role in shaping individual's response to PRT over the course of treatment. Future studies should seek to characterize how external environmental factors outside the clinical setting may interact with individual's biology, and better understand the geneenvironment interaction underlying differential profiles of treatment response.

\section{Limitations and future directions}

Current neuroimaging findings of PRT are limited to studies with small sample sizes consisting of high-functioning school-aged children with ASD. Intervention studies with small sizes may be more influenced by inter-participant variance, and making it questionable whether heterogeneity in mechanisms of change captured is clinically meaningful and can be generalized to a wider clinical population. ${ }^{68}$ Comparing effect sizes of therapeutic gains from both published and unpublished PRT studies, Sham and Smith ${ }^{69}$ found that reported therapeutic gains were much greater in published studies, suggesting possible publication bias in empirical evidence supporting PRT.$^{70}$ However, despite potential publication bias, the average reported effect size still demonstrated PRT to be an effective intervention, and did not significantly impact PRT's status as an evidencebased treatment for individuals with ASD. ${ }^{69}$ Nonetheless, future studies should evaluate whether existing neuroimaging findings may be replicated using larger sample sizes, as well as assess generalizability to other age groups, particularly younger children with ASD, to further expand the evidence surrounding PRT and investigate its effectiveness as an early intervention for children with ASD. Future studies should also include lower-functioning individuals with ASD, in order to further increase reliability of findings discussed in the present review.

In addition, no neuroimaging studies up to date have used an effective control group, nor conducted follow-up assessments to examine long-term treatment outcomes following termination of PRT treatment. ${ }^{71}$ From a developmental perspective, it is important to include either an age-matched waitlist control or a treatment-as-usual group in order to examine if functional neural changes cannot be fully explained by development alone, but are rather treatmentspecific effects in response to PRT. Future studies should also aim to adopt a longitudinal design with a follow-up period to examine whether changes in functional response and connectivity when processing social information may last beyond the course of PRT. In addition, there is a need to evaluate whether clinical gains and predictors of treatment are specific to PRT, as opposed to other modes of behavioral- and communication-based interventions for children with ASD. Few randomized controlled trials (RCTs) have been conducted up to date. ${ }^{72-74}$ Although two RCTs have shown that PRT was able to elicit greater gains in social communication skills $^{72}$ and reductions in disruptive behavior ${ }^{73}$ when compared to highly structured ABA, another RCT found no differences in overall therapeutic gains in spoken language skills when comparing PRT to pictorial naturalistic communication strategies. ${ }^{74}$ Future studies should seek to include comparative intervention as well as treatment-as-usual groups, to further evaluate whether predictors and underlying mechanisms of response may be specific to PRT, to continue address the question of treatment specificity.

Future studies can also examine broader changes in other areas of brain, both within and beyond that of the social brain network. For example, using a broader range of tasks that require subjects to utilize other skills acquired during PRT, such as effective downregulation during affectively salient situations (top-down versus bottom-up affect regulation within the vmPFC-amygdala circuit), and the change 
in motivation when engaging in social versus nonsocial interchanges (ventral versus dorsal striatal response) might help better characterize other neural correlates outside the social brain, further expanding the range of objective neural biomarkers to help monitor and track progress over the course of PRT.

Finally, future studies can also consider using other modes of neuroimaging techniques such as electrophysiology (EEG) and event-related potentials (ERPs), as well as eye tracking, to assess changes in social competency following PRT. Both EEG/ERP and eye tracking can be used to investigate social information processing at a better temporal resolution compared to fMRI, and may cross-validate and extend existing findings from fMRI studies. Furthermore, cost per experiment is considerably lower for EEG/ERP and eye-tracking studies, and may be considered in a more favorable light for larger-scale studies with more frequent time points of assessment to monitor changes over the course of PRT, as well as at longer-term follow-ups.

Taken together, current findings presented based on fMRI neuroimaging provide a foundation for feasibility of finding objective biomarkers to help better understand mechanisms of change and predictors of therapeutic response in young children with ASD. At present, the neural findings presented have been solely generated based on fMRI, which is leading the scientific field in relation to identification of biological markers of treatment response and prediction. However, due to the expense of fMRI neuroimaging technique, there is a greater need to continue interdisciplinary research to examine the degree of convergence on finding biomarkers that can help predict treatment response by using other more cost-effective neuroimaging tools such as EEG/ERP, and eye tracking to facilitate more widespread use of technology as a clinical tool. Adapting existing and developing novel paradigms compatible with alternative modes of neuroimaging tools can generate results that can enable researchers to crossvalidate against current fMRI results, to not only increase the robustness of biomarkers identified but also increase the scalability of such research tools to better serve a larger clinical population.

\section{Conclusion}

Recent studies using neuroimaging techniques mark a first step towards partitioning the underlying heterogeneous mechanisms of change driving improvements in social competency following PRT. The tight coupling between neural and behavioral changes observed over the course of PRT provides promising preliminary data to support the use of these neural regions involved in social processing as potential objective biomarkers to help predict and capture both the quality and magnitude of changes in social information processing throughout intervention. Such findings highlight that interdisciplinary research implementing neuroimaging techniques and qualitative behavioral measures can better aid the characterization of heterogeneous trajectories of change across multiple levels, ranging from behaviors to brain regional and circuitry level, which can be especially meaningful when used to address the issue of equifinality. By helping clinicians better understand how PRT may be eliciting positive therapeutic gains for each individual, as well as predicting who may be most likely to benefit from PRT, neuroimaging research serves as an important milestone along the journey towards the development of precision medicine for individuals with ASD. ${ }^{41}$

\section{Acknowledgments}

Funding for PRT in the authors' laboratory came from the Autism Science Foundation, Simons Foundation (\#383661), Women's Health Research at Yale University (\#1087045), Deitz Family, Esme Usdan and Family, and Dwek Family to $\mathrm{PV}$.

\section{Disclosure}

The authors report no conflicts of interest in this work.

\section{References}

1. Kanner L. Autistic disturbances of affective contact. Nerv Child. 1943;2: 217-250.

2. American Psychiatric Association. Diagnostic and Statistical Manual of Mental Disorders. 5th ed. Washington, DC: American Psychiatric Association; 2013.

3. Hartley SL, Sikora DM, McCoy R. Prevalence and risk factors of maladaptive behaviour in young children with autistic disorder. $J$ Intellect Disabil Res. 2008;52(10):819-829.

4. Skinner B. The Behavior of Organisms: An Experimental Analysis. Oxford: Appleton-Century; 1938.

5. Lovaas OI. Behavioral treatment and normal educational and intellectual functioning in young autistic children. J Consult Clin Psychol. 1987;55(1):3-9.

6. Vismara LA, Rogers SJ. Behavioral treatments in autism spectrum disorder: what do we know? Annu Rev Clin Psychol. 2010;6:447-468.

7. Virués-Ortega J. Applied behavior analytic intervention for autism in early childhood: meta-analysis, meta-regression and dose-response meta-analysis of multiple outcomes. Clin Psychol Rev. 2010;30(4): 387-399.

8. Maurice C, Green G, Foxx RM. Making a Difference: Behavioral Intervention for Autism. Austin, TX: Pro-Ed, Inc.; 2001.

9. Peters-Scheffer N, Didden R, Korzilius H, Sturmey P. A meta-analytic study on the effectiveness of comprehensive ABA-based early intervention programs for children with autism spectrum disorders. PubMed Health; 2011 [cited January 13, 2017]. Available from: https://www.ncbi.nlm. nih.gov/pubmedhealth/PMH0032150/. Accessed February 1, 2017. 
10. Smith T. What is evidence-based behavior analysis? Behav Anal. 2013;36(1):7-33.

11. Koegel LK, Ashbaugh K, Koegel RL. Pivotal response treatment. In: Lang R, Hancock TB, Singh NN, editors. Early Intervention for Young Children with Autism Spectrum Disorder (Evidence-Based Practices in Behavioral Health). Basel: Springer International Publishing; 2016 [cited September 15, 2016]:85-112. Available from: http://link.springer. com/chapter/10.1007/978-3-319-30925-5_4

12. Koegel RL, Egel AL. Motivating autistic children. J Abnorm Psychol. 1979;88(4):418-426.

13. Seligman ME, Maier SF. Failure to escape traumatic shock. $J$ Exp Psychol. 1967;74(1):1-9.

14. Steege MW, Mace FC, Perry L, Longenecker H. Applied behavior analysis: beyond discrete trial teaching. Psychol Sch. 2007;44(1):91-99.0

15. Lovaas OI, Koegel R, Simmons JQ, Long JS. Some generalization and follow-up measures on autistic children in behavior therapy. $J$ Appl Behav Anal. 1973;6(1):131-165.

16. Delprato DJ. Comparisons of discrete-trial and normalized behavioral language intervention for young children with autism. J Autism Dev Disord. 2001;31(3):315-325.

17. Verschuur R, Didden R, Lang R, Sigafoos J, Huskens B. Pivotal response treatment for children with autism spectrum disorders: a systematic review. Rev J Autism Dev Disord. 2013;1(1):34-61.

18. Koegel RL, Koegel LK, editors. Treatment of pivotal areas. In: The PRT Pocket Guide: Pivotal Response Treatment for Autism Spectrum Disorders. Baltimore, MD: Paul H. Brookes Publishing Co.; 2012:13-38.

19. Ventola PE, Oosting DR, Keifer CM, Friedman HE. Toward optimal outcome following pivotal response treatment: a case series. Yale J Biol Med. 2015;88(1):37-44.

20. Koegel RL, Dyer K, Bell LK. The influence of child-preferred activities on autistic children's social behavior. J Appl Behav Anal. 1987;20(3): 243-252.

21. Dunlap G, Koegel RL. Motivating autistic children through stimulus variation. J Appl Behav Anal. 1980;13(4):619-627.

22. Dunlap G. The influence of task variation and maintenance tasks on the learning and affect of autistic children. J Exp Child Psychol. 1984; 37(1):41-64.

23. Ventola P, Friedman H, Oosting D. Pivotal response treatment: case reports. Psychoanal Study Child. 2015;69:242-260.

24. Koegel RL, O'Dell M, Dunlap G. Producing speech use in nonverbal autistic children by reinforcing attempts. J Autism Dev Disord. 1988; 18(4):525-538.

25. Koegel LK, Koegel RL, Shoshan Y, McNerney E. Pivotal response intervention II: preliminary long-term outcome data. Res Pract Pers Sev Disabil. 1999;24(3):186-198.

26. Koegel LK, Carter CM, Koegel RL. Teaching children with autism self-initiations as a pivotal response. Top Lang Disord. 2003;23(2): 134-145.

27. Koegel LK, Koegel RL, Green-Hopkins I, Barnes CC. Brief report: question-asking and collateral language acquisition in children with autism. J Autism Dev Disord. 2009;40(4):509-515.

28. Koegel L, Singh A, Koegel R, Hollingsworth J, Bradshaw J. Assessing and improving early social engagement in infants. J Posit Behav Interv. 2014;16(2):69-80

29. Koegel LK, Koegel RL, Hurley C, Frea WD. Improving social skills and disruptive behavior in children with autism through self-management. J Appl Behav Anal. 1992;25(2):341-353.

30. Ventola PE, Yang D, Abdullahi SM, Paisley CA, Braconnier ML, Sukhodolsky DG. Brief report: reduced restricted and repetitive behaviors after pivotal response treatment. J Autism Dev Disord. 2016; 46(8):2813-2820.

31. Vismara LA, Lyons GL. Using perseverative interests to elicit joint attention behaviors in young children with autism theoretical and clinical implications for understanding motivation. J Posit Behav Interv. 2007; 9(4):214-228

32. Stahmer AC. Teaching symbolic play skills to children with autism using pivotal response training. J Autism Dev Disord. 1995;25(2):123-141.
33. Baron-Cohen S, Leslie AM, Frith U. Does the autistic child have a "theory of mind"? Cognition. 1985;21(1):37-46.

34. Baron-Cohen S. The autistic child's theory of mind: a case of specific developmental delay. J Child Psychol Psychiatry. 1989;30(2): 285-297.

35. Baron-Cohen S. Autism and symbolic play. Br J Dev Psychol. 1987; 5(2):139-148.

36. Baron-Cohen S. Theory of mind and autism: a review. Int Rev Res Ment Retard. 2000;23:169-184.

37. Jarrold C, Boucher J, Smith P. Symbolic play in autism: a review. J Autism Dev Disord. 1993;23(2):281-307.

38. Ventola P, Yang DY, Friedman HE, et al. Heterogeneity of neural mechanisms of response to pivotal response treatment. Brain Imaging Behav. 2015;9(1):74-88.

39. Venkataraman A, Yang DY, Dvornek N, et al. Pivotal response treatment prompts a functional rewiring of the brain among individuals with autism spectrum disorder. Neuroreport. 2016;27(14): 1081-1085.

40. Yang D, Pelphrey KA, Sukhodolsky DG, et al. Brain responses to biological motion predict treatment outcome in young children with autism. Transl Psychiatry. 2016;6(11):e948.

41. Insel TR. The NIMH Research Domain Criteria (RDoC) Project: precision medicine for psychiatry. Am J Psychiatry. 2014;171(4):395-397.

42. Cicchetti D, Rogosch FA. Equifinality and multifinality in developmental psychopathology. Dev Psychopathol. 1996;8(4):597-600.

43. Kaiser MD, Hudac CM, Shultz S, et al. Neural signatures of autism. Proc Natl Acad Sci U S A. 2010;107(49):21223-21228.

44. Voos AC, Pelphrey KA, Tirrell J, et al. Neural mechanisms of improvements in social motivation after pivotal response treatment: two case studies. J Autism Dev Disord. 2013;43(1):1-10.

45. Johansson G. Visual perception of biological motion and a model for its analysis. Percept Psychophys. 1973;14(2):201-211.

46. Klin A, Lin DJ, Gorrindo P, Ramsay G, Jones W. Two-year-olds with autism orient to non-social contingencies rather than biological motion. Nature. 2009;459(7244):257-261.

47. Simion F, Regolin L, Bulf H. A predisposition for biological motion in the newborn baby. Proc Natl Acad Sci US A. 2008;105(2):809-813.

48. Frith CD, Frith U. Interacting minds - a biological basis. Science. 1999;286(5445):1692-1695.

49. Dittrich WH, Troscianko T, Lea SE, Morgan D. Perception of emotion from dynamic point-light displays represented in dance. Perception. 1996;25(6):727-738.

50. Castelli F, Happé F, Frith U, Frith C. Movement and mind: a functional imaging study of perception and interpretation of complex intentional movement patterns. Neuroimage. 2000;12(3):314-325.

51. Castelli F, Frith C, Happé F, Frith U. Autism, Asperger syndrome and brain mechanisms for the attribution of mental states to animated shapes. Brain. 2002;125(Pt 8):1839-1849.

52. Hill EL, Frith U. Understanding autism: insights from mind and brain. Philos Trans R Soc Lond B Biol Sci. 2003;358(1430):281-289.

53. Lord C, Rutter M, DiLavore PC, Risi S, Gotham K, Bishop SL. Autism Diagnostic Observation Schedule, Second Edition (ADOS-2) Manual (Part I): Modules 1-4. Torrence, CA: Western Psychological Services; 2012.

54. Paslawski T. The Clinical Evaluation of Language Fundamentals, Fourth Edition (CELF-4): a review. Can J Sch Psychol. 2005;20(1-2): 129-134.

55. Pinkham AE, Hopfinger JB, Pelphrey KA, Piven J, Penn DL. Neural bases for impaired social cognition in schizophrenia and autism spectrum disorders. Schizophr Res. 2008;99(1-3):164-175.

56. Wyk BC, Hudac CM, Carter EJ, Sobel DM, Pelphrey KA. Action understanding in the superior temporal sulcus region. Psychol Sci. 2009;20(6):771-777.

57. Markram K, Markram H. The intense world theory - a unifying theory of the neurobiology of autism. Front Hum Neurosci. 2010;4:224.

58. Chevallier C, Kohls G, Troiani V, Brodkin ES, Schultz RT. The social motivation theory of autism. Trends Cogn Sci. 2012;16(4):231-239. 
59. Elsabbagh M, Mercure E, Hudry K, et al; BASIS Team. Infant neural sensitivity to dynamic eye gaze is associated with later emerging autism. Curr Biol. 2012;22(4):338-342.

60. Dawson G, Webb SJ, McPartland J. Understanding the nature of face processing impairment in autism: insights from behavioral and electrophysiological studies. Dev Neuropsychol. 2005;27(3):403-424.

61. Johnson MK, Raye CL, Mitchell KJ, Touryan SR, Greene EJ, Nolen-Hoeksema S. Dissociating medial frontal and posterior cingulate activity during self-reflection. Soc Cogn Affect Neurosci. 2006;1(1): 56-64.

62. Andrews-Hanna JR, Smallwood J, Spreng RN. The default network and self-generated thought: component processes, dynamic control, and clinical relevance. Ann N Y Acad Sci. 2014;1316:29-52.

63. Dayan E, Sella I, Mukovskiy A, et al. The default mode network differentiates biological from non-biological motion. Cereb Cortex. 2016;26(1):234-245.

64. Scott-Van Zeeland AA, Abrahams BS, Alvarez-Retuerto AI, et al. Altered functional connectivity in frontal lobe circuits is associated with variation in the autism risk gene CNTNAP2. Sci Transl Med. 2010; 2(56):56ra80.

65. Schultz RT, Gauthier I, Klin A, et al. Abnormal ventral temporal cortical activity during face discrimination among individuals with autism and Asperger syndrome. Arch Gen Psychiatry. 2000;57(4):331-340.

66. Sherer MR, Schreibman L. Individual behavioral profiles and predictors of treatment effectiveness for children with autism. J Consult Clin Psychol. 2005;73(3):525-538.
67. Schreibman L, Stahmer AC, Barlett VC, Dufek S. Brief report: toward refinement of a predictive behavioral profile for treatment outcome in children with autism. Res Autism Spectr Disord. 2009;3(1):163-172.

68. Ioannidis JP. Why most published research findings are false. PLoS Med. 2005;2(8):e124.

69. Sham E, Smith T. Publication bias in studies of an applied behavioranalytic intervention: an initial analysis. J Appl Behav Anal. 2014;47(3): 663-678.

70. Easterbrook PJ, Berlin JA, Gopalan R, Matthews DR. Publication bias in clinical research. Lancet. 1991;337(8746):867-872.

71. Cadogan S, McCrimmon AW. Pivotal response treatment for children with autism spectrum disorder: a systematic review of research quality. Dev Neurorehabil. 2015;18(2):137-144.

72. Mohammadzaheri F, Koegel LK, Rezaee M, Rafiee SM. A randomized clinical trial comparison between pivotal response treatment (PRT) and structured applied behavior analysis (ABA) intervention for children with autism. J Autism Dev Disord. 2014;44(11):2769-2777.

73. Mohammadzaheri F, Koegel LK, Rezaei M, Bakhshi E. A randomized clinical trial comparison between pivotal response treatment (PRT) and adult-driven applied behavior analysis (ABA) intervention on disruptive behaviors in public school children with autism. J Autism Dev Disord. 2015;45(9):2899-2907.

74. Schreibman L, Stahmer AC. A randomized trial comparison of the effects of verbal and pictorial naturalistic communication strategies on spoken language for young children with autism. J Autism Dev Disord. 2014;44(5):1244-1251.
Neuropsychiatric Disease and Treatment

\section{Publish your work in this journal}

Neuropsychiatric Disease and Treatment is an international, peerreviewed journal of clinical therapeutics and pharmacology focusing on concise rapid reporting of clinical or pre-clinical studies on a range of neuropsychiatric and neurological disorders. This journal is indexed on PubMed Central, the 'PsycINFO' database and CAS,

\section{Dovepress}

and is the official journal of The International Neuropsychiatric Association (INA). The manuscript management system is completely online and includes a very quick and fair peer-review system, which is all easy to use. Visit http://www.dovepress.com/testimonials.php to read real quotes from published authors. 